\title{
The story of adult veno-venous extracorporeal membrane oxygenation (ECMO) in Sri Lanka; a case report of leptospirosis-associated severe pulmonary haemorrhagic syndrome (SPHS)
}

\author{
Harischandra DVT ${ }^{1}$, Withanaarachchi WAUK ${ }^{2}$, Firmin RK $^{3}$ \\ ${ }^{1}$ Cardiothoracic Unit, ${ }^{2}$ Neonatal Intensive Care Unit, Teaching Hospital Karapitiya, Galle, Sri Lanka. \\ ${ }^{3}$ University Hospitals of Leicester, Leicester, United Kingdom. \\ Correspondence: Dr. Tolusha Harischandra \\ e-mail: tolushah@gmail.com \\ (D) https://orcid.org/0000-0001-9791-5431
}

\section{Introduction}

Successful extracorporeal membrane oxygenation (ECMO) in Sri Lanka was first reported in 2017 in a neonate in Galle (1). Galle is endemic to leptospirosis, one of the emerging infections with a high case fatality rate in Sri Lanka (2). The estimated annual caseload of leptospirosis in Sri Lanka from 2008 to 2015 was 10,423 (2). Recently, there have been reports describing the use of ECMO in adults with severe leptospirosis-related pulmonary involvement in the medical literature (3). Physicians in our institution, concerned with the escalating number of deaths, particularly in young, fit men having leptospirosis-related severe pulmonary haemorrhagic syndrome (SPHS), approached our ECMO team to discuss the possibility of using ECMO in such adults.

This is the first report of adult veno-venous ECMO performed in Sri Lanka. It illustrates how medical evidence, even in the form of sporadic case reports, combined with multidisciplinary discussion can save lives and bring about significant change in a medical system.

\section{Case presentation}

A 27-year-old, 55kg, previously healthy shopkeeper from Elpitiya was admitted to the local hospital with four days of fever, headache, arthralgia, myalgia and conjunctival suffusion. There was a history of contact with mud following the recent floods. He was transferred to Teaching Hospital Karapitiya, Galle with a clinical diagnosis of leptospirosis with pulmonary haemorrhage for "plasmapheresis and further management".

On admission, he was in severe respiratory distress with tachypnoea and an oxygen saturation $\left(\mathrm{SaO}_{2}\right)$ of $75 \%$, and needed inotropes to maintain his blood pressure. A few hours later, he collapsed and was intubated and ventilated. There was bleeding into the endotracheal tube and bilateral consolidation with alveolar (cotton-wool) shadows on the chest radiograph, signifying pulmonary haemorrhage (Figure 1a).

$\mathrm{He}$ was prescribed intravenous $\mathrm{C}$ penicillin $2 \mathrm{MU}$ 6 hourly, ceftriaxone $1 \mathrm{~g} 12$ hourly, immunoglobulin $20 \mathrm{~g}$ daily and methyl prednisolone $1 \mathrm{~g}$ daily. Therapeutic plasma exchange (TPE) was commenced on the first day.

Over the next two days he became oliguric and thrombocytopaenic but remained normokalaemic. There were $\mathrm{T}$ inversions on the inferolateral leads of the electrocardiogram, and mild global hypokinesia with $45-50 \%$ ejection fraction on the echocardiogram. The second cycle of TPE was done. The hypoxia and respiratory acidosis worsened despite high ventilator settings: Peak/ mean inspiratory pressures 49/24mmHg; Positive End Expiratory Pressures $12 \mathrm{mmHg}$; Fraction of inspired oxygen $100 \% . \quad \mathrm{PaO}_{2} 58 \mathrm{mmHg} ; \mathrm{pCO}_{2}$ $79 \mathrm{mmHg} ; \mathrm{pH} 7.2$; bicarbonate $20 \mathrm{mg} / \mathrm{dl}$. The Murray score was four. At this point our ECMO team was contacted. Although eligible for ECMO, the only ECMO system available was already being used for a paediatric patient. As the adult was 
rapidly deteriorating, a decision was made to use the back-up ECMO pump and console without the heater cooler and blender, which was left on the paediatric patient. As an adult pump head was not available, we used a re-sterilized one from our demonstration kit.

Informed assent was obtained from the relations. The patient was cannulated percutaneously using $20 \mathrm{~F}$ drainage and $18 \mathrm{~F}$ return cannulae (Figure $1 \mathrm{~b}$ ) in the left femoral and right internal jugular veins respectively and placed on veno-venous ECMO (Figure 2).
This allowed blood from the IVC to be drained out of the body, oxygenated by the oxygenator and pumped back via the centrifugal pump into the right atrium.

The patient stabilized over the next few hours: His $\mathrm{pH}$ improved to 7.4 with $\mathrm{pCO}_{2}$ of $45 \mathrm{mmHg}$; $\mathrm{SaO}_{2}$ 88-90\% with ECMO flows of approximately $4 \mathrm{~L} / \mathrm{min}$. The ventilator settings were gradually reduced to rest settings allowing the inotropes to be weaned off. Standard heparinisation protocols were followed with a target activated clotting time of 180 - 200 seconds.

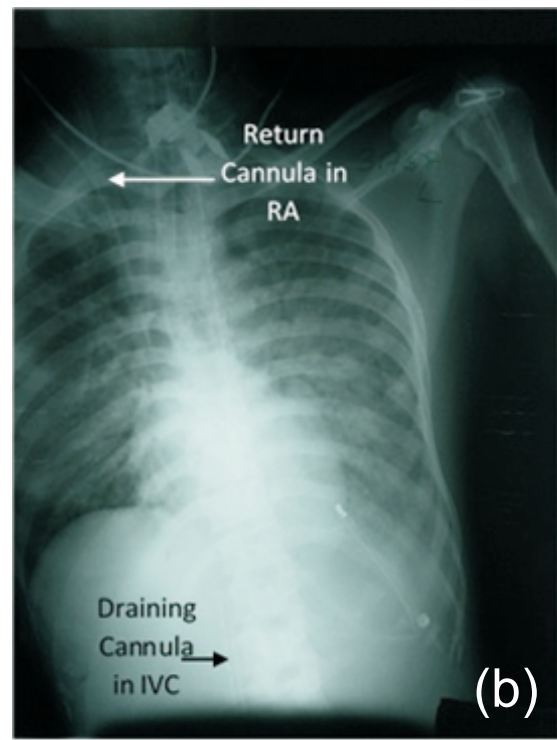

Figure 1: Chest radiographs at presentation (a) and after 10 days on ECMO (b)

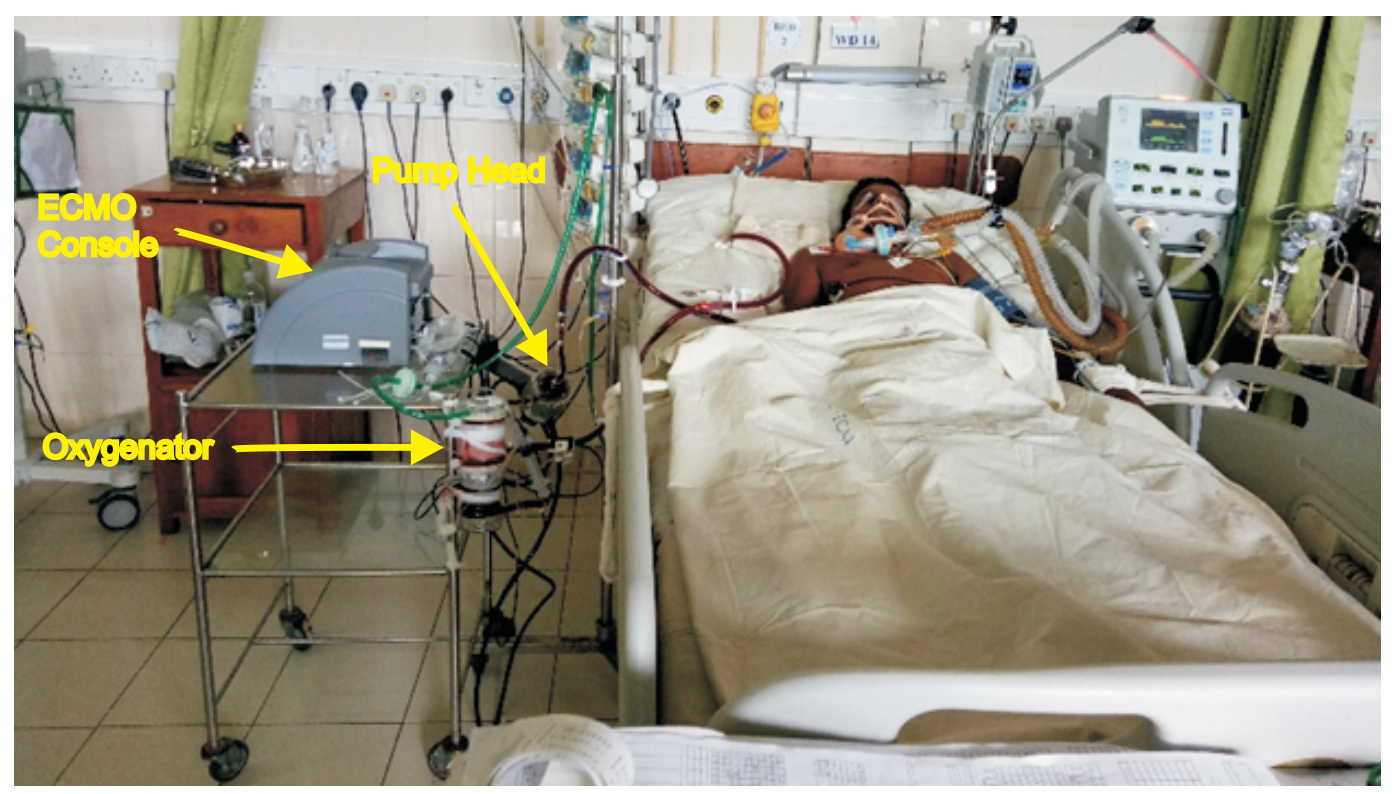

Figure 2: Patient on a minimalistic ECMO circuit 
The microscopic agglutination test (MAT), performed using the genus-specific Patoc strain, sent on the $6^{\text {th }}$ day of illness became strongly positive. As the patient's respiratory and haemodynamic parameters remained stable, treatment continued with immunoglobulin, methylprednisolone and two more cycles of TPE. Nosocomial lung infection with multidrug resistant Acinetobacter and Pseudomonas species had set in needing intravenous imipenem $\lg 8$ hourly, teicoplanin $400 \mathrm{mg}$ daily, nebulized amikacin $500 \mathrm{mg}$ daily and oral doxycycline $100 \mathrm{mg}$ 12 hourly over the next two weeks.

After 254 hours of ECMO, clinical and radiological parameters improved (Figure 1b) and he was weaned off ECMO successfully and decannulated. A tracheostomy was performed the next day. He was ventilated for 22 days and discharged from ICU after 30 days. His critical illness gradually resolved and neuropathy due to prolonged immobilization subsided to allow discharge five days later. At clinic review, he was well and described an out-of-body experience during the illness. Two years later, he remains well and fully functional.

\section{Discussion}

ECMO uses cardiopulmonary bypass technology in the intensive care setting. It is not a treatment but rather a support technique for cardio-respiratory function. This allows time for treatment (such as antibiotics, steroids, plasmapheresis) or simply the body's immunity to act in a patient who would otherwise not be alive.

The first successful ECMO in the world was performed in 1972, in an adult with acute respiratory distress syndrome due to trauma (4). Forty-five years later, the first adult ECMO in Sri Lanka was performed for leptospirosis-associated SPHS. Leptospirosis is not a common condition in the west where there is greater availability of ECMO than in the tropics where leptospirosis is endemic. Therefore, only a few case reports are available in the world literature as described by Arokianathan, et al. from Leicester (5), Umei, et al. from Tokyo (3) and Schmalzle, et al. from Hawaii (6), all showing the benefit of ECMO in leptospirosis. It was awareness of such reports that encouraged local Physicians to request Cardiothoracic Surgeons to extend their initial experience in neonatal ECMO to the adult arena. Today, ECMO is part of the therapeutic armamentarium for all age groups in
Sri Lanka and the final frontier in managing severe respiratory and/ or cardiac failure not amenable to conventional treatment.

Written informed consent was obtained from the patient for publication of this case report with photographs.

\section{Acknowledgements}

We thank Chalice Medical Ltd., UK for the loan of their ECMO machine as well as Drs. Wimalasiri Uluwattage (consultant physician), Nalaka Herath (consultant nephrologist) and Saliya Wijenayake (consultant anaesthetist) for encouraging us to perform our first adult.

\section{References}

1. Harischandra, DVT, Withanaarachchi K, Kumara MT, Firmin RK. Mechanical cardio-pulmonary support in critical care; early experience with Extracorporeal Membrane Oxygenation (ECMO) in Sri Lanka. Galle Medical Journal, 2017; 22(2): 30-34. DOI: http://doi.org/10.4038/gmj.v22i2.7973.

2. Warnasekara J, Koralegedara I, Agampodi S. Estimating the burden of leptospirosis in Sri Lanka; a systematic review. BMC Infectious Diseases, Dec 1 2019; 19(1): 119. https://doi.org/10.1186/s12879-018-3655-y

3. Umei N, Ichiba S. A Case of Leptospirosis-Associated Severe Pulmonary Hemorrhagic Syndrome Successfully Treated with Venovenous Extracorporeal Membrane Oxygenation. Case Rep Crit Care, 2017. Article ID: 5369267. DOI: https://doi.org/10.1155/2017/5369267.

4. Hill JD, O'Brien TG, Murray JJ, et al. Prolonged extracorporeal oxygenation for acute post-traumatic respiratory failure (shock-lung syndrome). Use of the bramson membrane lung. The New England Journal of Medicine, 1972; 286: 629-634. DOI: 10.1056/ nejm197203232861204.

5. Arokianathan D, Trower K, Pooboni S, Sosnowski A, Moss P, Thaker H. Leptospirosis: a case report of a patient with pulmonary haemorrhage successfully managed with extra corporeal membrane oxygenation. Journal of Infection, $2005 \mathrm{Feb}$; 50(2): 158-62.

6. Schmalzle SA, Tabatabai A, Mazzeffi $\mathrm{M}$, et al. Recreational 'mud fever': Leptospira interrogans induced diffuse alveolar hemorrhage and severe acute respiratory distress syndrome in a U.S. Navy seaman following 'mudrun' in Hawaii. IDCases, 2019; 15: e00529. Published 2019 Mar 23. DOI: 10.1016/j.idcr.2019.e00529. 